\title{
A WEAK CONVERGENCE APPROACH TO HYBRID LQG PROBLEMS WITH INFINITE CONTROL WEIGHTS
}

\author{
G. GEORGE YIN ${ }^{1}$ \\ Wayne State University \\ Department of Mathematics \\ Detroit, MI 48202 US \\ JIONGMIN YONG ${ }^{2}$ \\ Fudan University \\ Laboratory of Mathematics for Nonlinear Sciences \\ Department of Mathematics \\ Shanghai 200433 China
}

(Received June, 2001; Revised December, 2001)

\begin{abstract}
This work is concerned with a class of hybrid LQG (linear quadratic Gaussian) regulator problems modulated by continuous-time Markov chains. In contrast to the traditional LQG models, the systems have both continuous dynamics and discrete events. In lieu of a model with constant coefficients, these coefficients vary with time and exhibit piecewise constant behavior. At any time $t$, the system follows a stochastic differential equation in which the coefficients take one of the $m$ possible configurations where $m$ is usually large. The system may jump to any of the possible configurations at random times. Further, the control weight in the cost functional is allowed to be indefinite. To reduce the complexity, the Markov chain is formulated as singularly perturbed with a small parameter. Our effort is devoted to solving the limit problem when the small parameter tends to zero via the framework of weak convergence. Although the limit system is still modulated by a Markov chain, it has a much smaller state space and thus, much reduced complexity.
\end{abstract}

Key words: LQC Problem, Hybrid Control, Markov Chains, Weak Convergence, Indefinite Control Weight.

AMS subject classifications: 93E20, 60F05.

${ }^{1}$ The research of this author was supported in part by the National Science Foundation under grants DMS9877090 and DMS-9971608.

${ }^{2}$ The research of this author was supported by the NSFC under grant 79790130, the National Distinguished Youth Science Foundation of China under grant 19725106, the Science Foundation of MOE of China under grant 2000024605, and the Cheung Kong Scholars Programme of MOE of China. Part of the work was done while this author was visiting Wayne State University. 


\section{Introduction}

This work is concerned with hybrid LQG (linear quadratic Gaussian) problems which are a class of LQG regulator problems modulated by continuous-time Markov chains. Working with the finite-time horizon $\left[0, T_{0}\right]$ for some $T_{0}>0$, the dynamic system is given by

$$
\left\{\begin{aligned}
d x(t) & =\{A(\alpha(t)) x(t)+B(\alpha(t)) u(t)\} d t \\
& +\{C(\alpha(t)) x(t)+D(\alpha(t)) u(t)\} d w(t), t \in\left[0, T_{0}\right], \\
x(0) & =x \\
\alpha(0) & =\alpha
\end{aligned}\right.
$$

where $A(\cdot), B(\cdot), C(\cdot), D(\cdot)$ are matrix-valued functions with appropriate dimensions, $w(\cdot)$ is a one-dimensional standard Brownian motion, and $\alpha(\cdot)$ is a continuous-time Markov chain. Our objective is to minimize the cost function

$$
\begin{aligned}
& J(x, \alpha ; u(\cdot))= \\
& \quad E\left(\int_{0}^{T_{0}}\left\{x(t)^{T} M(\alpha(t)) x(t)+u(t)^{T} N(\alpha(t)) u(t)\right\} d t+x\left(T_{0}\right)^{T} H x\left(T_{0}\right)^{T} H x\left(T_{0}\right)\right)
\end{aligned}
$$

over $u(\cdot)$ in the set of all admissible controls (see the next section), subject to (1.1), where $z^{T}$ denotes the transpose of $z$. There are at least two main features in the above problem:

(1) a continuous-time Markov chain drives the coefficients of the system and affects the cost functional, and the matrices $M(\cdot), N(\cdot)$ and $H$ are indefinite (not necessarily nonnegative definite).

This work is motivated by the recent developments of robust controls [1], stochastic optimal controls of LQG regulators with indefinite control weight cost $[2-4,15]$, and hybrid control systems $[12,16]$. We analyze a class of uncertain linear systems in which the system coefficients are subject to discrete event disturbances. The model includes both continuous dynamics of diffusion type and jump processes; see also [7, 8] and others for references of jump linear systems. The study of controls of LQG has received much attention because one can often approximate a nonlinear system by a linear or piecewise linear system. The inclusion of Markov pure jump processes reflects our effort to address the robustness issue when random fluctuations are taken into consideration. Unlike a fixed system, these system coefficients switch among a number of possible configurations. In practical terms, we are dealing with a system of equations in lieu of a single equation. In our formulation, although the Markov chain has a finite state space, the state space may be very large in many applications (e.g., stochastic networks). Thus, the total number of equations to be solved is large resulting in much computational difficulty. To reduce the complexity, we note that among the transition of states, some vary rapidly and others change slowly. To take advantage of the contrasts between the rates of changes, we introduce a small parameter $\epsilon>0$. Thus, the Markov chain becomes $\alpha(t)=\alpha^{\epsilon}(t)$. The different rates of change are conveniently modeled using the small parameter $\epsilon$ in the generator of the Markov chain which leads to singularly perturbed Markovian systems (see the recent results in [12-14]). The class of LQG problems then becomes one involving singularly perturbed jump processes. The problem is similar to that of [16], but the control weights in the cost functional are indefinite.

In contrast to the approach used in [16], in this paper we do not work with the HJB (Hamilton-Jacobi-Bellman) equations. Instead, our effort is to use weak convergence methods 
to derive limit control problems. Because it is purely probabilistic, we can deal with timevarying generators with fewer restrictions. For instance, if the singularly perturbed Markov chain under consideration includes only recurrent states, we only require that the generators be bounded and Borel measurable (no smoothness is required). Our main effort is devoted to showing that there is an associated limit problem corresponding to the problem involving singularly perturbed Markov chain with indefinite control weights, obtained by aggregating the states in each weakly irreducible class into one state. We derive the limit system that is still an LQG problem modulated by a continuous-time Markov chain with indefinite control weights where the total number of configurations for the limit problem is substantially reduced.

After determining the limit systems, the rest of the paper is arranged as follows. Section 2 presents the formation of the problem together with the conditions needed in the subsequent asymptotic analysis. Section 3 is devoted to the weak convergence of the dynamic systems and the convergence of the cost function. For ease of presentation, our focus in Sections 2 and 3 is on the dominating part of the generator corresponding to a Markov chain with $\ell$ weakly irreducible classes. Section 4 gives the results for the case that additional transient states are also included. Finally, further remarks are made in Section 5.

Before proceeding, a word of notation is in order. For simplicity, we use $K$ to denote a generic positive constant whose value may change for different appearances; the convention $K+K=K$ and $K K=K$ is understood. We use $f_{x}$ to denote the gradient of $f$ with respect to the variable $x$ and use $|\cdot|$ to denote a norm in an appropriate space.

\section{Formulation}

\subsection{Singularly Perturbed Markov Chains}

Let $(\Omega, \mathcal{F}, \mathbf{P})$ be a complete probability space. For the small valued parameter $\epsilon>0$, let $\alpha^{\epsilon}(\cdot)$ be a Markov chain defined on $(\Omega, \mathcal{F}, \mathbf{P})$ that takes on values in a finite state space $\mathcal{M}$ of $m$ points. Suppose that the generator $Q^{\epsilon}(\cdot)$ of $\alpha^{\epsilon}(\cdot)$ is given by

$$
Q^{\epsilon}(t)=\frac{1}{\epsilon} \widetilde{Q}(t)+\widehat{Q}(t), \quad t \geq 0,
$$

where $\widetilde{Q}(\cdot)=\left(\widetilde{q}_{i j}(\cdot)\right)$ and $\widehat{Q}(\cdot)=\left(\widehat{q}_{i j}(\cdot)\right)$ are $m \times m$ measurable matrix-valued functions defined on $\left[0, T_{0}\right]$ with $0<T_{0}<\infty$.

Recall that for a nonstationary (time inhomogeneous) Markov chain $\gamma(t)$, a matrix-valued function $Q(\cdot):\left[0, T_{0}\right] \mapsto \mathcal{M}$ with $Q(t)=\left(q_{i j}(t)\right), t \geq 0$, is a generator of $\gamma(t)$ if

(1) $\quad q_{i j}(t)$ is Borel measurable for all $i, j \in \mathcal{M}$ and $0 \leq t \leq T_{0}$, and $q_{i j}(t)$ is uniformly bounded;

(2) $\quad q_{i j}(t) \geq 0$ for $j \neq i$ and $q_{i i}(t)=-\sum_{j \neq i} q_{i j}(t), t \geq 0$;

for a real-valued function $g$ defined on $\mathcal{M}$

$$
g(\gamma(t))=\int_{0}^{t} Q(s) g(\cdot)(\gamma(s)) d s
$$

is a martingale. The notation $Q(s) g(\cdot)(i)$ is meant to be

$$
Q(s) g(\cdot)(i)=\sum_{j \in \mathcal{M}} q_{i j}(s) g(j)=\sum_{\substack{j \in \mathcal{M} \\ j \neq i}} q_{i j}(s)(g(j)-(i)) \quad i \in \mathcal{M} .
$$


We say that $Q(t)$ or the corresponding Markov chain is weakly irreducible if the system of equations

$$
\mu(t) Q(t)=0, \quad \mu(t) \mathbb{I}_{m}=1
$$

has a unique nonnegative solution where $\mathbb{I}_{j}$ denotes a $j$-dimensional column vector with all components equal to 1 . The unique solution $\mu(t)$ is termed a quasi-stationary distribution.

The model (2.1) is motivated by the consideration of time-scale separation. In lieu of twotime scales, multi-time scales can be considered with minor modifications but with more complicated notation. By a first glance, it seems that the generator (2.1) is confined to fixed forms of functions $\widetilde{Q}(\cdot)$ and $\widehat{Q}(\cdot)$. That is, the forms of decomposition or partition of $\widetilde{Q}(\cdot)$ into $\widetilde{Q}^{i}(\cdot)$ and the form of $\widehat{Q}(\cdot)$ do not vary with respect to time. This restriction can, however, be removed by introducing another level of time-varying function. That is, assume the structure of (2.1) changes with respect to time $t$, but the change is also Markovian. To do so, one can define a combined process $\gamma^{\epsilon}(t)=\left(\beta(t), \alpha^{\epsilon}(t)\right)$, whereas $\beta(t)$ itself is also a continuous-time Markov chain with finite state space $\mathcal{M}_{\beta}$ (with $m_{\beta}$ states). Then the generator (2.1) for the process $\alpha^{\epsilon}(t)$ will take $m_{\beta}$ many possible forms. At any time $t$, with $\beta(t)=\beta \in \mathcal{M}_{\beta}=\left\{1, \ldots, m_{\beta}\right\}$ dictated by the Markov chain $\beta(t)$, the generator of $\alpha^{\epsilon}(t)$ takes the form (2.1) with $\widetilde{Q}(t)=\widetilde{Q}_{\beta}(t)$ and $\widehat{Q}(t)=\widehat{Q}_{\beta}(t)$. It can be shown that the combined process again has a generator that is of the form (2.1). Thus the model we are considering is sufficiently general. Note that it is also possible to consider a system in which $\beta(\cdot)$ is replaced by a diffusion process. In that case, one obtains a switching diffusion process; for some of the recent work along this line, see [6] and the references therein. For other related results and references concerning singularly perturbed Markov chains, see [1214].

It is clear that the dominating part of the generator $Q^{\epsilon}(\cdot)$ is given by $\widetilde{Q}(\cdot)$ due to the presence of the scaling $1 / \epsilon$. We first concentrate on the case of inclusion of $\ell$ classes of weakly irreducible states only. (The case regarding inclusion of additional transient states will be discussed in Section 4.) Assume that $\widetilde{Q}(\cdot)$ takes the form

$$
\widetilde{Q}(t)=\left(\begin{array}{cccc}
\widetilde{Q}^{1}(t) & & & \\
& \widetilde{Q}^{2}(t) & & \\
& & \ddots & \\
& & & \widetilde{Q}^{\ell}(t)
\end{array}\right) \stackrel{\operatorname{def}}{=} \operatorname{diag}\left(\widetilde{Q}^{1}(t), \ldots, \widetilde{Q}^{\ell}(t)\right), t \geq 0 .
$$

Hereafter, $\operatorname{diag}\left(Z^{1}, \ldots, Z^{\ell}\right)$ denotes a block diagonal matrix with matrix entries $Z^{1}, \ldots, Z^{\ell}$ with the appropriate dimensions. We will need the following assumption.

(A1) Functions $\widehat{Q}(\cdot):\left[0, T_{0}\right] \mapsto \mathbb{R}^{m \times m}$ and $\widetilde{Q}^{i}(\cdot):\left[0, T_{0}\right] \mapsto \mathbb{R}^{m_{i} \times m_{i}}(i=1, \ldots, \ell)$ are Borel measurable and bounded, and are themselves generators of some Markov chains. Moreover, for each $t \in\left[0, T_{0}\right]$, and each $i=1, \ldots, \ell, \widetilde{Q}^{i}(t)$ is weakly irreducible.

In view of $\widetilde{Q}(t)$, the state space admits the decomposition:

$$
\begin{gathered}
\mathcal{M}=\left\{\zeta_{11}, \ldots, \zeta_{1 m_{1}}\right\} \cup\left\{\zeta_{21}, \ldots, \zeta_{2 m_{2}}\right\} \cup \ldots \cup\left\{\zeta_{\ell 1}, \ldots, \zeta_{\ell m_{\ell}}\right\} \\
=\mathcal{M}_{1} \cup \mathcal{M}_{2} \cup \ldots \cup \mathcal{M}_{\ell}
\end{gathered}
$$

such that $\mathcal{M}_{i} \equiv\left\{\zeta_{i 1}, \ldots, \zeta_{i m_{i}}\right\}$ is the state space of a Markov chain with the generator $\widetilde{Q}^{i}(\cdot)$. By examining the structure of $\widetilde{Q}(t)$, we can aggregate the states in each $\mathcal{M}_{i}$ as one state. 
Define $\bar{\alpha}^{\epsilon}(t)=i$ if $\alpha^{\epsilon}(t) \in \mathcal{M}_{i}$. The following results hold; proofs can be found in Theorems 7.28 and 7.30 of [12] respectively.

Lemma 2.1: Suppose (A1) is satisfied. Then the following assertions hold.

(i) For $i=1, \ldots, \ell, j=1, \ldots, m_{i}$, and for all $\rho(\cdot) \in L^{2}\left[0, T_{0}\right]$, then as $\epsilon \rightarrow 0$,

$$
\int_{0}^{T_{0}}\left[P\left(\alpha^{\epsilon}(t)=\zeta_{i j}\right)-\nu_{j}^{i}(t) \theta(t)\right] \rho(t) d t \rightarrow 0
$$

where $\theta(t)=\left(\theta_{1}(\theta), \ldots, \theta_{\ell}(t)\right) \in \mathbb{R}^{1 \times \ell}$ is the solution of

$$
\begin{gathered}
\dot{\theta}(t)=\theta(t) \bar{Q}(t), \theta(0)=p_{0} \operatorname{diag}\left(\mathbb{I}_{m_{1}}, \ldots, \mathbb{I}_{m_{\ell}}\right), \\
\bar{Q}(t)=\operatorname{diag}\left(\nu^{1}(t), \ldots, \nu^{\ell}(t)\right) \widehat{Q}(t) \operatorname{diag}\left(\mathbb{I}_{m_{1}}, \ldots, \mathbb{I}_{m_{\ell}}\right),
\end{gathered}
$$

$\nu^{i}(t)$ is the quasi-stationary distribution corresponding to $\widetilde{Q}^{i}(t)$, for $i=1, \ldots, \ell$, and $p_{0}=\left(P\left(\alpha^{\epsilon}(0)=\zeta_{i j}\right)\right) \in \mathbb{R}^{1 \times m}$ is the initial distribution of the Markov chain.

(ii) As $\epsilon \rightarrow 0, \bar{\alpha}^{\epsilon}(\cdot)$ converges weakly to $\bar{\alpha}(\cdot)$ in $D\left[0, T_{0}\right]$, where $D\left[0, T_{0}\right]$ is the space real-valued functions that are right continuous have left limits endowed with the Skorohod topology. The limit $\bar{\alpha}(t)$ is a Markov chain with generator $\bar{Q}(t)$ given by (2.6) and state space $\overline{\mathcal{M}}=\{1, \ldots, \ell\}$.

Remark 2.2: Various asymptotic properties of singularly perturbed Markov chains can be found in [12]. We will not dwell on them here.

\subsection{Formulation}

In addition to the complete probability space $(\Omega, \mathcal{F}, \mathbf{P})$ and the singularly perturbed Markov chain $\alpha^{\epsilon}(\cdot)$ given above, suppose that a one-dimensional standard Brownian motion $w(\cdot)$ is defined on $(\Omega, \mathcal{F}, \mathbf{P})$. Let $\left(\mathcal{F}_{t}^{w}\right\}_{t \geq 0}$ and $\left\{\mathcal{F}_{t}^{\epsilon}\right\}_{t \geq 0}$ be the natural filtrations generated by $w(\cdot)$ and by $\left(w(\cdot), \alpha^{\epsilon}(\cdot)\right)$, respectively. Let $\mathcal{U}\left[0, T_{0}\right]$

and $\mathcal{U}^{\epsilon}\left[0, T_{0}\right]$ be the set of all processes $u:\left[0, T_{0}\right] \times \Omega \rightarrow \mathbb{R}^{r}$ such that

$E \int_{0}^{T_{0}}|u(t)|^{2} d t<\infty$ and $\left\{\mathcal{F}_{t}^{w}\right\}_{t \geq 0^{-}}$(resp. $\left.\left\{\mathcal{F}^{\epsilon}\right\}_{t \geq 0^{-}}\right)$adapted. We consider the following LQG problem.

Problem LQG ${ }^{\epsilon}$ : Minimize

$$
\begin{gathered}
J^{\epsilon}(x, \alpha ; u(\cdot))=E\left(\int_{0}^{T_{0}}\left\{x^{\epsilon}(t)^{T} M\left(\alpha^{\epsilon}(t)\right) x^{\epsilon}(t)+u(t)^{T} N\left(\alpha^{\epsilon}(t)\right) u(t)\right\} d t\right. \\
\left.+x^{\epsilon}\left(T_{0}\right)^{T} H x^{\epsilon}\left(T_{0}\right)\right),
\end{gathered}
$$

over $u(\cdot) \in \mathcal{U}^{\epsilon}\left[0, T_{0}\right]$ subject to

$$
\left\{\begin{array}{l}
d x^{\epsilon}(t)=\left[A\left(\alpha^{\epsilon}(t)\right) x^{\epsilon}(t)+B\left(\alpha^{\epsilon}(t)\right) u(t)\right] d t \\
+\left[C\left(\alpha^{\epsilon}(t)\right) x^{\epsilon}(t)+D\left(\alpha^{\epsilon}(t)\right) u(t)\right] d w(t), \quad t \in\left[0, T_{0}\right], \\
\quad x^{\epsilon}(0)=x \\
\quad \alpha^{\epsilon}(0)=\alpha .
\end{array}\right.
$$


In the above, $x^{\epsilon}(\cdot)$ is an $\mathbb{R}^{r}$-valued state process, $u(\cdot)$ is an $\mathbb{R}^{n}$-valued control process, $A(\cdot), B(\cdot), C(\cdot), D(\cdot), M(\cdot), N(\cdot)$ are matrix-valued functions of proper dimensions defined on $\mathcal{M}$, and $H$ is a matrix. We would like to study the limit of the above problem as $\epsilon \rightarrow 0$. To this end, we need the following additional assumption.

(A2) The following conditions hold.

(a) $\quad A, C, M: \mathcal{M} \rightarrow \mathbb{R}^{r \times r}, B, D: \mathcal{M} \rightarrow \mathbb{R}^{r \times n}, N: \mathcal{M} \rightarrow \mathbb{R}^{n \times n}$ are matrix-valued functions, $M(\iota)$ and $N(\iota)$ are symmetric for each $\iota=1,2, \ldots, m$, and $H \in \mathbb{R}^{r \times r}$ is symmetric.

(b) The Brownian motion $w(\cdot)$ and the Markov chain $\alpha^{\epsilon}(\cdot)$ are independent.

(c) The problem given by (2.7) and (2.8) is uniquely solvable. The control $u(t)$ given in a feedback form is

$$
u(t)=-\Psi(t, \iota) x^{\epsilon}(t), \text { for each } \iota \in \mathcal{M} \text { and } t \in\left[0, T_{0}\right] .
$$

where $\Psi(\cdot)$ is a suitable function.

Remark 2.3: The solvability of LQG problems with indefinite control weights is treated in [2] for deterministic coefficient cases and was further dealt with in [3, 4] for random coefficient cases. For further discussions related to this and the associated forward-backward stochastic differential equations, we refer the reader to $[3,4,15]$ and the references therein. For the problem we are considering, we can work with each $\iota \in \mathcal{M}$ in a way similar to equations (6) and (7) of [16] due to the piecewise constant nature of the coefficients and the independence between the Brownian motion and the Markov chains. See also [12, Sections A.4 and A.5, pp. 309-325] for discussions on HJB equations and optimal controls for jump processes.

For ease of presentation, we have assumed the solvability of the hybrid LQG problem. If $\widetilde{Q}(t)=\widetilde{Q}$ and $\widehat{Q}(t)=\widehat{Q}$, independent of time, a sufficient condition for (A2) part $(c)$ can be given as follows: For each $\iota=1, \ldots, m$, the Riccati equation

$$
\left\{\begin{array}{c}
\dot{P}^{\epsilon}(t, \iota)+P^{\epsilon}(t, \iota) A(\iota)+A(\iota)^{T} P^{\epsilon}(t, \iota)+C(\iota)^{T} P^{\epsilon}(t, \iota) C(\iota)+M(\iota) \\
-\left[P^{\epsilon}(t, \iota) B(\iota)+C(\iota)^{T} P^{\epsilon}(t, \iota) D(\iota)\right]\left[N(\iota)+D(\iota)^{T} P^{\epsilon}(t, \iota) D(\iota)\right]^{-1} \\
\times\left[B(\iota)^{T} P^{\epsilon}(t, \iota)+D(\iota)^{T} P^{\epsilon}(t, \iota)+D(\iota)^{T} P^{\epsilon}(t, \iota) C(\iota)\right] \\
+Q^{\epsilon}(t) P^{\epsilon}(t, \cdot)(\iota)=0, t \in\left[0, T_{0}\right] \\
P^{\epsilon}\left(t_{0}, \iota\right)=H,
\end{array}\right.
$$

admits a unique solution. Moreover, the function $\Psi(\cdot, \iota)$ given in (2.9) can be specified as

$$
\begin{gathered}
\Psi(t, \iota)=\left[N(\iota)+D(\iota)^{T} P^{\epsilon}(t, \iota) D(\iota)\right]^{-1}\left[B(\iota)^{T} P^{\epsilon}(t, \iota)+D(\iota)^{T} P^{\epsilon}(t, \iota) C(\iota)\right], \\
t \in\left[0, T_{0}\right],
\end{gathered}
$$

which is well-defined and bounded. [Note that for time-varying $\widetilde{Q}(\cdot)$ and $\widehat{Q}(\cdot)$, similar Riccati equations for $P^{\epsilon}(t, \iota)$ can also be derived, but require much more involved notation.] In fact, by combining and modifying the approaches in [2] and [16] together with the use of [12, Appendix], (A2) part $(c)$ holds if the system of Riccati equations admits a solution. Then, we may further proceed to derive the bounds on $P^{\epsilon}(t, \iota)$ and obtain its convergence to a limit that is a solution of a limit Riccati equation. Nevertheless, such an approach is not the focus of this paper; we assume the solvability instead.

Another alternative determining the solvability of LQG problems is to consider $\left\{u^{\epsilon}(\cdot)\right\}$ and use the topology of weak convergence in the space of square integrable functions for the control sequence. One can then work with the triple $\left(x^{\epsilon}(\cdot), \bar{\alpha}^{\epsilon}(\cdot), u^{\epsilon}(\cdot)\right)$ and proceed with the weak convergence analysis. Except for not using relaxation, the process is similar to 
working with a relaxed control formulation (see $[9,12])$. As far as the weak convergence part is concerned, the argument is similar to the argument in this paper.

Lemma 2.4: Let $(A 1)$ and $(A 2)$ hold. For any $(x, \alpha) \in \mathbb{R}^{r} \times \mathcal{M}$, let $u(\cdot) \in \mathcal{U}^{\epsilon}\left[0, T_{0}\right]$, then the state process $x^{\epsilon}(\cdot)$ satisfies the following estimates:

$$
\underset{t \in\left[0, T_{0}\right]}{E \sup _{0}} E\left|x^{\epsilon}(t)\right|^{2} \leq K \text {, and } E \sup _{t \in\left[0, T_{0}\right]} E\left|x^{\epsilon}(t)\right|^{4} \leq K .
$$

Proof: The proof is standard; for example, see [15].

In what follows, we use $E_{t}^{\epsilon}$ and $E_{t}^{w}$ to denote the corresponding conditional expectations with respect to $\mathcal{F}_{t}^{\epsilon}$ and $\mathcal{F}_{t}^{w}$, respectively.

Lemma 2.5: Assume $(A 1)$ and $(A 2)$ hold. Suppose $g(x)$ is a bounded $\mathbb{R}^{r}$-valued function together with bounded derivatives. Denote $Z_{i j}^{\epsilon}(t)=Z_{i j}\left(t, \alpha^{\epsilon}(t)\right)$ with

$$
Z_{i j}(t, \alpha)=I_{\left\{\alpha=\zeta_{i j}\right\}}-\nu_{j}^{i}(t) I_{\left\{\alpha \in \mathcal{M}_{i}\right\}} .
$$

Then for any $i=1, \ldots, l, j=1, \ldots, m_{i}$,

$$
\sup _{0<t \leq T} E\left|\int_{0}^{t} g\left(x^{\epsilon}(\tau)\right)^{T} x^{\epsilon}(\tau) Z_{i j}^{\epsilon}(\tau) d \tau\right|^{2} \rightarrow 0 \text {, as } \epsilon \rightarrow 0,
$$

where $x^{\epsilon}(\cdot)$ is the solution of $(2.8)$.

Proof: Pick a $\Delta \in(0,1)$. For any $t \in\left[0, T_{0}\right]$, partition $[0, t]$ into subintervals of equal length $\epsilon^{1-\Delta}$ (without loss of generality, assume that $N=t / \epsilon^{1-\Delta}$ is an integer, otherwise, we can always take its integer part.) Denote the partition boundaries by $t_{k}=k \epsilon^{1-\Delta}$ for $0 \leq k \leq N+1$. Define

$$
\begin{gathered}
\widetilde{g}^{\epsilon}(\tau)=\left\{\begin{array}{cc}
g\left(x^{\epsilon}(0)\right), & \tau \in\left[0, t_{2}\right), \\
g\left(x^{\epsilon}\left(t_{k-1}\right)\right), & \tau \in\left[t_{k}, t_{k+1}\right), 2 \leq k \leq N+1,
\end{array}\right. \\
\widetilde{x}^{\epsilon}(\tau)=\left\{\begin{array}{cc}
x^{\epsilon}(0), & \tau \in\left[0, t_{2}\right), \\
x^{\epsilon}\left(t_{k-1}\right), & \tau \in\left[t_{k}, t_{k+1}\right), 2 \leq k \leq N+1 .
\end{array}\right.
\end{gathered}
$$

In the above, we have assumed a "perfect" division of intervals. Generally, the length of the last interval in the subdivision may be shorter than the others so a modification may be necessary. However, for notational simplicity, we ignore the end-interval modification here and henceforth.

It follows that

$$
\begin{gathered}
E\left|\int_{0}^{t} g\left(x^{\epsilon}(\tau)\right)^{T} x^{\epsilon}(\tau) Z_{i j}^{\epsilon}(\tau) d \tau\right|^{2} \leq K E\left|\int_{0}^{t}\left[g\left(x^{\epsilon}(\tau)\right)-\widetilde{g}^{\epsilon}(\tau)\right]^{T} x^{\epsilon}(\tau) Z_{i j}^{\epsilon}(\tau) d \tau\right|^{2} \\
+\left.K E\left|\int_{0}^{t} \widetilde{g}^{\epsilon}(\tau)^{T}\left[x^{\epsilon}(\tau)-\widetilde{x}^{\epsilon}(\tau)\right] Z_{i j}^{\epsilon}(\tau) d \tau\right|\right|^{2}
\end{gathered}
$$




$$
+K E\left|\int_{0}^{t} \widetilde{g}^{\epsilon}(\tau)^{T} \widetilde{x}^{\epsilon}(\tau) Z_{i j}^{\epsilon}(\tau) d \tau\right|^{2} .
$$

We first show that the first two terms on the right-hand side of (2.16) do not contribute anything to the limit. For the next to the last term of (2.16), noting the boundedness of $Z_{i j}^{\epsilon}(\tau)$ and $\widetilde{g}^{\epsilon}(\tau)$, we have

$$
\begin{gathered}
E\left|\int_{0}^{t} \widetilde{g}^{\epsilon}(\tau)^{T}\left[x^{\epsilon}(\tau)-\widetilde{x}^{\epsilon}(\tau)\right] Z_{i j}^{\epsilon}(\tau) d \tau\right|^{2} \\
\leq K \int_{0}^{t} E\left|x^{\epsilon}(\tau)-\widetilde{x}^{\epsilon}(\tau)\right|^{2} d \tau \\
=K \sum_{k=2} \int_{t_{k}}^{t_{k+1}} E\left|x^{\epsilon}(\tau)-x^{\epsilon}\left(t_{k-1}\right)\right|^{2} d \tau+K \int_{0}^{t_{2}} E\left|x^{\epsilon}(\tau)-x^{\epsilon}(0)\right|^{2} d \tau \\
\leq K \sum_{k=2}^{N} \int_{t_{k}}^{t_{k+1}}\left(\tau-t_{k-1}\right) d \tau+K \int_{0}^{t_{2}} \tau d \tau \\
=O\left(\epsilon^{1-\Delta}\right) \rightarrow 0 \text { as } \epsilon \rightarrow 0 .
\end{gathered}
$$

Note in the above that we have used Lemma 2.4 and the fact that for $\tau \in\left[t_{k}, t_{k+1}\right)$,

$$
E\left|x^{\epsilon}(\tau)-x^{\epsilon}\left(t_{k-1}\right)\right|^{2} \leq K\left(\tau-t_{k-1}\right) .
$$

In a similar way, using Lemma 2.4, we also have

$$
E\left|x^{\epsilon}(\tau)\right|^{2}<\infty, \text { and } E\left|x^{\epsilon}(\tau)-x^{\epsilon}\left(t_{k-1}\right)\right|^{4} \leq K\left(\tau-t_{k-1}\right)^{2} .
$$

Thus, for the first term on the right-hand side of (2.16), using the boundedness of $E\left|x^{\epsilon}(\tau)\right|^{4}$ and the Lipschitz continuity of $g(\cdot)$, we have

$$
\begin{gathered}
E\left|\int_{0}^{t}\left[g\left(x^{\epsilon}(\tau)\right)-\widetilde{g}^{\epsilon}(\tau)\right]^{T} x^{\epsilon}(\tau) Z_{i j}^{\epsilon}(\tau) d \tau\right|^{2} \\
\leq K E \int_{0}^{t}\left|g\left(x^{\epsilon}(\tau)\right)-\widetilde{g}^{\epsilon}(\tau)\right|^{2}\left|x^{\epsilon}(\tau)\right|^{2} d \tau \\
\leq K \int_{0}^{t} E^{1 / 2}\left|g\left(x^{\epsilon}(\tau)\right)-\widetilde{g}^{\epsilon}(\tau)\right|{ }^{4} E^{1 / 2}\left|x^{\epsilon}(\tau)\right|^{4} d \tau
\end{gathered}
$$




$$
\begin{gathered}
=K \sum_{k=2}^{N} \int_{t_{k}}^{t_{k+1}} \sqrt{E\left|x^{\epsilon}(\tau)-x^{\epsilon}\left(t_{k-1}\right)\right|^{4}} d \tau+K \int_{0}^{t_{2}} \sqrt{E \mid x^{\epsilon}(\tau)-x^{\epsilon}(0){ }^{4}} d \tau \\
=O\left(\epsilon^{2(1-\Delta)}\right) \rightarrow 0 \text { as } \epsilon \rightarrow 0 .
\end{gathered}
$$

In view of (2.17) and (2.18), we need only examine the last term in (2.16).

$$
\begin{array}{r}
E\left|\int_{0}^{t} \widetilde{g}^{\epsilon}(\tau)^{T} \widetilde{x}^{\epsilon}(\tau) Z_{i j}^{\epsilon}(\tau) d \tau\right|^{2} \\
=2 E \int_{0}^{t} \int_{0}^{v}\left(\widetilde{x}^{\epsilon}(\tau)\right)^{T} \widetilde{g}^{\epsilon}(\tau) \widetilde{g}^{\epsilon}(\tau)^{T} \widetilde{x}^{\epsilon}(\tau) \Phi^{\epsilon}(v, \tau) d \tau d v,
\end{array}
$$

where

$$
\Phi^{\epsilon}(v, \tau)=\Phi_{1}^{\epsilon}(v, \tau)+\Phi_{2}^{\epsilon}(v, \tau),
$$

with

$$
\begin{gathered}
\Phi_{1}^{\epsilon}(v, \tau)=I_{\left\{\alpha^{\epsilon}(\tau)=\zeta_{i j}, \alpha^{\epsilon}(v)=\zeta_{i j}\right\}}-\nu_{j}^{i}(v) I_{\left\{\alpha^{\epsilon}(v) \in \mathcal{M}_{i}, \alpha^{\epsilon}(\tau)=\zeta_{i j}\right\}} \\
\Phi_{2}^{\epsilon}(v, \tau)=-\nu_{j}^{i}(\tau) I_{\left\{\alpha^{\epsilon}(\tau) \in \mathcal{M}_{i}, \alpha^{\epsilon}(v)=\zeta_{i j}\right\}}+\nu_{j}^{i}(\tau) \nu_{j}^{i}(v) I_{\left\{\alpha^{\epsilon}(v) \in \mathcal{M}_{i}, \alpha^{\epsilon}(\tau) \in \mathcal{M}_{i}\right\}}
\end{gathered}
$$

Similar to [12, Theorem 7.29], it can be shown

$$
\int_{0}^{t} \int_{0}^{v} E\left(\Phi_{i}^{\epsilon}(v, \tau) \mid \alpha^{e}(s)\right) d \tau d v \rightarrow 0 \text { as } \epsilon \rightarrow 0, \text { for } i=1,2 .
$$

By the independence of $w(\cdot)$ and $\alpha^{\epsilon}(\cdot)$, for $s \leq \tau \leq v$,

$$
\begin{gathered}
\int_{0}^{t} \int_{0}^{v} E\left(\Phi^{\epsilon}(v, \tau) \mid \mathcal{F}_{s}^{\epsilon}\right)=\int_{0}^{t} \int_{0}^{v} E\left(\Phi^{\epsilon}(v, \tau) \mid \alpha^{\epsilon}(s), s \leq \tau\right) d \tau d v \\
=\iint_{0}^{t} \int_{0}^{v} E\left(\Phi^{\epsilon}(v, \tau) \mid \alpha^{\epsilon}(\tau)\right) d \tau d v \\
=\int_{0}^{t} \int_{0}^{v} E\left(\Phi_{1}^{\epsilon}(v, \tau) \mid \alpha^{\epsilon}(\tau)\right) d \tau d v+\int_{0}^{t} \int_{0}^{v} E\left(\Phi_{2}^{\epsilon}(v, \tau) \mid \alpha^{\epsilon}(\tau)\right) d \tau d v \\
\rightarrow 0 \text { as } \epsilon \rightarrow 0 .
\end{gathered}
$$

Using the boundedness of $\widetilde{g}^{\epsilon}(\cdot)$ and interchanging the orders of integrations lead to 


$$
\begin{gathered}
E \int_{0}^{t} \int_{0}^{v} \widetilde{x}^{\epsilon}(\tau)^{T} \widetilde{g}^{\epsilon}(\tau) \widetilde{g}^{\epsilon}(v)^{T} \widetilde{x}^{\epsilon}(v) \Phi^{\epsilon}(v, \tau) d \tau d v \\
=E \int_{0}^{t} \int_{\tau}^{t} \widetilde{x}^{\epsilon}(\tau)^{T} \widetilde{g}^{\epsilon}(\tau) \widetilde{g}^{\epsilon}(v)^{T} \widetilde{x}^{\epsilon}(v, \tau) d v d \tau \\
E \int_{0}^{t} \widetilde{x}^{\epsilon}(\tau)^{T} \widetilde{g}^{\epsilon}(\tau) E_{\tau}^{\epsilon}\left(\sum_{k \leq N+1} \int_{t_{k}}^{t_{k+1}} \widetilde{g}^{\epsilon}(v)^{T} \widetilde{x}^{\epsilon}(v) \Phi(v, \tau) d v\right) d \tau \\
=E \int_{0}^{t} \widetilde{x}^{\epsilon}(\tau)^{T} \widetilde{g}^{\epsilon}(\tau) E_{\tau}^{\epsilon}\left(\sum_{k \leq N+1} \int_{t_{k}}^{t_{k+1}} \widetilde{g}^{\epsilon}(v)^{T} \widetilde{x}^{\epsilon}(v) E\left(\Phi^{\epsilon}(v, \tau) \mid \mathcal{F}_{t_{k-1}}\right) d v\right) d \tau
\end{gathered}
$$

Examining the estimates obtained thus far, they also hold uniformly in $t \in\left[0, T_{0}\right]$. Therefore, the lemma is proved.

Focusing on the feedback control of the form given in (A2), we use $u^{\epsilon}(t)=$ $-\Psi\left(\alpha^{\epsilon}(t)\right) x^{\epsilon}(t)$. As mentioned above, the hybrid feature presents many difficulties. If we proceed with an approach of dynamic programming, we need to solve a system of HJB equations (in fact, $m$ equations). Since $m$ represents the number of states of the Markov chain, which is usually very large, the computational task could become a formidable one. Even with today's computing power, the amount of computation may be too large to be feasible. It is thus necessary to find viable alternatives. Using the idea of decomposition and aggregation (see related discussions in [10]), we aggregate the states in each weakly irreducible class as one state. This leads to an associated limit problem or reduced problem, which is an average with respect to the quasi-stationary distribution of the singularly perturbed Markov chain. Our approach is purely probabilistic. Rather than working with HJB equations, we concentrate on probability measures and derive the limit results via weak convergence methods.

Associated with $\mathcal{P}^{\epsilon}$, there is a limit problem. We denote it as follows.

$\mathcal{P}^{0}$ Problem: Minimize

$J(x, \bar{\alpha})=$

$$
E\left(\int_{0}^{T_{0}} x(t)^{T}\left[\bar{M}(\alpha(t))+\overline{\Psi(t, \bar{\alpha}(t))^{T} N(\bar{\alpha}(t)) \Psi(t, \bar{\alpha}(t))}\right] x(t) d t+x\left(T_{0}\right)^{T} H x\left(T_{0}\right)\right)
$$

subject to

$$
\begin{gathered}
d x(t)=\overline{A(\bar{\alpha}(t))-B(\bar{\alpha}(t)) \Psi(t, \bar{\alpha}(t))} d t+\bar{\Sigma}(t, \bar{\alpha}(t)) x(t) d w(t), \\
x(0)=x
\end{gathered}
$$




$$
\bar{\alpha}(0)=\bar{\alpha}
$$

where for each $i \in \overline{\mathcal{M}}=\{1, \ldots, \ell\}$,

$$
\begin{aligned}
& \overline{\Psi(t, i)^{T} N(i) \Psi(t, i)}=\sum_{i=1}^{\ell} \sum_{j=1}^{m_{i}}\left[\Psi\left(t, \zeta_{i j}\right)^{T} N\left(\zeta_{i j}\right) \Psi\left(t, \zeta_{i j}\right)\right] \nu_{j}^{i}(t), \\
& \overline{A(i)-B(i) \Psi(t, i)}=\sum_{i=1}^{\ell} \sum_{j=1}^{m_{i}}\left[A\left(\zeta_{i j}\right)-B\left(\zeta_{i j}\right) \Psi\left(t, \zeta_{i j}\right)\right] \nu_{j}^{i}(t),
\end{aligned}
$$

where $\bar{\Sigma}(t, i)$ satisfies

$$
\begin{aligned}
\bar{\Sigma}(t, i) \bar{\Sigma}(t, i)^{T} & = \\
\sum_{i=1}^{\ell} & \sum_{j=1}^{m_{i}}\left[C\left(\zeta_{i j}\right)-D\left(\zeta_{i j}\right) \Psi\left(t, \zeta_{i j}\right)\right]\left[C\left(\zeta_{i j}\right)-D\left(\zeta_{i j}\right) \Psi\left(t, \zeta_{i j}\right)\right]^{T} \nu_{j}^{i}(t) .
\end{aligned}
$$

Remark 2.6: Note that for a suitable function $F(\cdot), \bar{F}(\cdot)$ represents the average with respect to the quasi-stationary distributions $\nu^{i}(t)$ for $i=1, \ldots, \ell$.

\section{Weak Convergence Analysis}

This section is divided into three parts: tightness, weak convergence of $\left(x^{\epsilon}(\cdot), \bar{\alpha}^{\epsilon}(\cdot)\right)$, and the convergence of the cost functions.

\subsection{Tightness}

To prove the tightness of $x^{\epsilon}(\cdot)$, using the a priori bound in Lemma 2.4, we have

$$
\begin{aligned}
\left|x^{\epsilon}(t+s)-x^{\epsilon}(t)\right|^{2} \leq 2\left|\int_{t}^{t+s}\left[A\left(\alpha^{\epsilon}(v)\right)-B\left(\alpha^{\epsilon}(v)\right) \Psi\left(v, \alpha^{\epsilon}(v)\right)\right] x^{\epsilon}(v) d v\right|^{2} \\
+2\left|\int_{t}^{t+s}\left[C\left(\alpha^{\epsilon}(v)\right)-D\left(\alpha^{\epsilon}(v)\right) \Psi\left(v, \alpha^{\epsilon}(v)\right)\right] x^{\epsilon}(v) d w(v)\right|^{2}
\end{aligned}
$$

By virtue of Lemma 2.4 and the assumptions on $A(\iota), B(\iota)$, and $\Psi(v, \iota)$, it is easily seen that

$$
E E_{t}^{\epsilon}\left|\int_{t}^{t+s}\left[A\left(\alpha^{\epsilon}(v)\right)-B\left(\alpha^{\epsilon}(v)\right) \Psi\left(v, \alpha^{\epsilon}(v)\right)\right] x^{\epsilon}(v) d v\right|^{2} \leq K s^{2}
$$

and, by using the assumptions on $C(\iota), D(\iota)$, the independence of $w(\cdot)$ and $\alpha^{\epsilon}(\cdot)$, and the well-known results of Ito calculus, 


$$
\begin{gathered}
E E_{t}^{\epsilon}\left|\int_{t}^{t+s}\left[C\left(\alpha^{\epsilon}(v)\right)-D\left(\alpha^{\epsilon}(v)\right) \Psi\left(v, \alpha^{\epsilon}(v)\right)\right] x^{\epsilon}(v) d w(v)\right|^{2} \\
\leq E E_{t}^{\epsilon} \int_{t}^{t+s}\left|C\left(\alpha^{\epsilon}(v)\right)-D\left(\alpha^{\epsilon}(v)\right) \Psi\left(v, \alpha^{\epsilon}(v)\right)\right|^{2}\left|x^{\epsilon}(v)\right|^{2} d v \\
\leq K s .
\end{gathered}
$$

Thus, (3.1) and (3.2) imply

$$
\lim _{s \rightarrow 0} \limsup _{\epsilon \rightarrow 0} E E_{t}^{\epsilon}\left|x^{\epsilon}(t+s)-x^{\epsilon}(t)\right|^{2}=0 .
$$

The tightness criteria (see [5, Section 3.8, p. 132]) yield that $\left\{x^{\epsilon}(\cdot)\right\}$ is tight in $D^{r}\left[0, T_{0}\right]$, where $D^{r}\left[0, T_{0}\right]$ denotes the space of $\mathbb{R}^{r}$-valued functions that are right continuous, have left limits, and are endowed with the Skorohod topology (see [5, p. 122]). Moreover, the weak convergence of $\bar{\alpha}^{\epsilon}(\cdot)$ implies that $\left\{\bar{\alpha}^{\epsilon}(\cdot)\right\}$ is also tight. Furthermore, the well-known Cramér-Wold device implies that $\left\{\left(x^{\epsilon}(\cdot), \bar{\alpha}^{\epsilon}(\cdot)\right\}\right.$ is tight in $D^{r+1}\left[0, T_{0}\right]$. We summarize the above discussion with the following theorem.

Theorem 3.1: Under $(A 1)$ and $(A 2),\left\{x^{\epsilon}(\cdot)\right\}$ is tight in $D^{r}\left[0, T_{0}\right] . \quad$ Moreover, $\left\{\left(x^{\epsilon}(\cdot), \bar{\alpha}^{\epsilon}(\cdot)\right)\right\}$ is tight in $D^{r+1}\left[0, T_{0}\right]$.

Remark 3.2: In fact, the full capacity of assumptions (A1) and (A2) is not needed. What is essential is that the continuous-time Markov chain has a finite state space with bounded and measurable generators, $A(\iota), B(\iota)$, etc. are all well defined, and that $w(\cdot)$ and $\alpha^{\epsilon}(\cdot)$ are independent.

\subsection{Weak Convergence}

In view of Prohorov's theorem, the previously established tightness allows us to extract weakly convergent subsequences. By virtue of the martingale average techniques (see [9]), we proceed th characterize the limit process.

Theorem 3.3: Assume that the conditions of Theorem 3.1 are satisfied. Then $\left(x^{\epsilon}(\cdot), \bar{\alpha}^{\epsilon}(\cdot)\right)$ converges weakly to $(x(\cdot), \bar{\alpha}(\cdot))$ such that $x(\cdot)$ is the solution of the stochastic differential equation (2.20).

Proof: We use the martingale averaging techniques to resolve the problem. Let us consider the pair $\left(x^{\epsilon}(\cdot), \bar{\alpha}^{\epsilon}(\cdot)\right)$. The sequence $\left\{\left(x^{\epsilon}(\cdot), \bar{\alpha}^{\epsilon}(\cdot)\right)\right\}$ is tight. Extract a weakly convergent subsequence and still denote it by $\left\{x^{\epsilon}(\cdot), \bar{\alpha}^{\epsilon}(\cdot)\right\}$. By virtue of the Skorohod representation (see [5, p. 102]), we may assume without changing notation that $\left(x^{\epsilon}(\cdot), \bar{\alpha}^{\epsilon}(\cdot)\right)$ converges to $(x(\cdot), \bar{\alpha}(\cdot))$ w.p.l and the convergence is uniform on any bounded time interval. To obtain the desired convergence result, we need to show the weak limit $(x(\cdot), \bar{\alpha}(\cdot))$ is the solution of a martingale problem with generator $\mathcal{L}$, where

$$
\begin{aligned}
& \mathcal{L} f(x, i)= \\
& \quad f_{x}(x, i)^{T}[\overline{A(i)-B(i) \Psi(t, i)}] x+\frac{1}{2} x^{T} \bar{\Sigma}(t, i)^{T} f_{x x}(x, i) \bar{\Sigma}(t, i) x+\bar{Q}(t) f(x, \cdot)(i),
\end{aligned}
$$

for each $i \in \overline{\mathcal{M}}=\{1, \ldots, \ell\}, f(\cdot)$ is a suitable function, and 


$$
\bar{Q}(t) f(x, \cdot)(i)=\sum_{j \in \overline{\mathcal{M}}} \bar{q}_{i j}(t) f(x, j)=\sum_{\substack{j \in \overline{\mathcal{M}} \\ j \neq i}} \bar{q}_{i j}(t)(f(x, j)-f(x, i)) .
$$

It suffices to show that for each $i \in \overline{\mathcal{M}}$, any positive integer $\kappa$, any $f(\cdot, i) \in C_{0}^{2}$ (the collection of $C^{2}$ functions with compact support), any bounded and continuous functions $h_{\iota}(\cdot, i)$ with $\iota \leq \kappa, t, s, t_{\iota} \geq 0$ and $t_{\iota} \leq t \leq t+s$, the following equation holds:

$$
\begin{gathered}
E \prod_{\iota=1}^{\kappa} h_{\iota}\left(x\left(t_{\iota}\right), \bar{\alpha}\left(t_{\iota}\right)\right)(f(x(t+s), \bar{\alpha}(t+s))-f(x(t), \bar{\alpha}(t)) \\
\left.-\int_{t}^{t+s} \mathcal{L} f(x(v), \bar{\alpha}(v)) d v\right)=0 .
\end{gathered}
$$

To begin, we consider the process indexed by $\epsilon$. With the $f(\cdot)$ chosen above, define

$$
f^{0}(x, \alpha)=\sum_{i=1}^{\ell} f(x, i) I_{\left\{\alpha \in \mathcal{M}_{i}\right\}} \text { for each } \alpha \in \mathcal{M}_{i} .
$$

Since the joint process $\left(x^{\epsilon}(\cdot), \alpha^{\epsilon}(\cdot)\right)$ is Markov with generator $\mathcal{L}^{\epsilon}$ given by

$$
\begin{gathered}
\mathcal{L}^{\epsilon} f^{0}(x, \alpha)=f_{x}^{0}(x, \alpha)^{T}[A(\alpha)-B(\alpha) \Psi(t, \alpha)] x \\
+\frac{1}{2} x^{T}[C(\alpha)-D(\alpha) \Psi(t, \alpha)]^{T} f_{x x}^{0}(x, \alpha)[C(\alpha)-D(\alpha) \Psi(t, \alpha)] x+Q^{\epsilon}(t) f^{0}(x, \cdot)(\alpha)
\end{gathered}
$$

for each $\alpha \in \mathcal{M}$,

$$
f^{0}\left(x^{\epsilon}(t+s), \alpha^{\epsilon}(t+s)\right)-f^{0}\left(x^{\epsilon}(t), \alpha^{\epsilon}(t)\right)-\int_{t}^{t+s} \mathcal{L}^{\epsilon} f^{0}\left(x^{\epsilon}(v), \alpha^{\epsilon}(v)\right) d v
$$

is a martingale. Thus,

$$
\begin{gathered}
E \prod_{\iota=1}^{\kappa} h_{\iota}\left(x^{\epsilon}\left(t_{\iota}\right), \bar{\alpha}^{\epsilon}\left(t_{\iota}\right)\right)\left[f^{0}\left(x^{\epsilon}(t+s), \alpha^{\epsilon}(t+s)\right)-f^{0}\left(x^{\epsilon}(t), \alpha^{\epsilon}(t)\right)\right. \\
\left.-\int_{t}^{t+s} \mathcal{L}^{\epsilon} f^{0}\left(x^{\epsilon}(v), \alpha^{\epsilon}(v)\right) d v\right]=0 .
\end{gathered}
$$

By the weak convergence of $\left(x^{\epsilon}(\cdot), \bar{\alpha}^{\epsilon}(\cdot)\right)$ to $(x(\cdot), \bar{\alpha}(\cdot))$, the definition (3.6), and the Skorohod representation,

$$
E \prod_{\iota=1}^{\kappa} h_{\iota}\left(x^{\epsilon}\left(t_{\iota}\right), \bar{\alpha}^{\epsilon}\left(t_{\iota}\right)\right)\left(f^{0}\left(x^{\epsilon}(t+s), \alpha^{\epsilon}(t+s)\right)-f^{0}\left(x^{\epsilon}(t), \alpha^{\epsilon}(t)\right)\right)
$$




$$
\rightarrow E \prod_{\iota=1}^{\kappa} h_{\iota}\left(x\left(t_{\iota}\right), \bar{\alpha}\left(t_{\iota}\right)\right)(f(x(t+s), \bar{\alpha}(t+s))-f(x(t), \bar{\alpha}(t))) \text { as } \epsilon \rightarrow 0 .
$$

To proceed, note that since $t_{\iota} \leq t, h_{\iota}\left(x^{\epsilon}\left(t_{\iota}\right), \bar{\alpha}\left(t_{\iota}\right)\right)$ is $\mathcal{F}_{t}^{\epsilon}$-measurable and

$$
\begin{aligned}
& E \prod_{\iota=1}^{\kappa} h_{\iota}\left(x^{\epsilon}\left(t_{\iota}\right), \bar{\alpha}^{\epsilon}\left(t_{\iota}\right)\right)\left(\int_{t}^{t+s} \mathcal{L}^{\epsilon} f^{0}\left(x^{\epsilon}(v), \bar{\alpha}(v)\right) d v\right) \\
= & E \prod_{\iota=1}^{\kappa} h_{\iota}\left(x^{\epsilon}\left(t_{\iota}\right), \bar{\alpha}^{\epsilon}\left(t_{\iota}\right)\right)\left(E_{t}^{\epsilon} \int_{t}^{t+s} \mathcal{L}^{\epsilon} f^{0}\left(x^{\epsilon}(v), \alpha^{\epsilon}(v)\right) d v\right),
\end{aligned}
$$

where $E_{t}^{\epsilon}$ denotes the conditioning on the $\sigma$-algebra generated by $\mathcal{F}_{t}^{\epsilon}$. The expression

$E \prod_{\iota=1}^{\kappa} h_{\iota}\left(x^{\epsilon}\left(t_{\iota}\right), \bar{\alpha}^{\epsilon}\left(t_{\iota}\right)\right)\left(E_{t}^{\epsilon} \int_{t}^{t+s} f_{x}^{0}\left(x^{\epsilon}(v), \alpha^{\epsilon}(v)\right)^{T}\left[A\left(\alpha^{\epsilon}(v)\right)-B\left(\alpha^{\epsilon}(v)\right) \Psi\left(v, \alpha^{\epsilon}(v)\right)\right] x^{\epsilon}(v) d v\right)$

can be rewritten as

$$
\begin{aligned}
& E \prod_{\iota=1}^{\kappa} h_{\iota}\left(x^{\epsilon}\left(t_{\iota}\right), \bar{\alpha}^{\epsilon}\left(t_{\iota}\right)\right) \\
& \quad \times\left[\sum_{i=1}^{\ell} \sum_{j=1}^{m_{i}} \int_{t}^{t+s} f_{x}^{0}\left(x^{\epsilon}(v), \zeta_{i j}\right)^{T}\left[A\left(\zeta_{i j}\right)-B\left(\zeta_{i j}\right) \Psi\left(v, \zeta_{i j}\right)\right] x^{\epsilon}(v) I_{\left\{\alpha^{\epsilon}(v)=\zeta_{i j}\right\}} d v\right] \\
& =E \prod_{\iota=1}^{\kappa} h_{\iota}\left(x^{\epsilon}\left(t_{\iota}\right), \bar{\alpha}^{\epsilon}\left(t_{\iota}\right)\right) \\
& \quad \times\left\{\sum_{i=1}^{\ell} \sum_{j=1}^{m_{i}} \int_{t}^{t+s} f_{x}\left(x^{\epsilon}(v), i\right)^{T}\left[A\left(\zeta_{i j}\right)-B\left(\zeta_{i j}\right) \Psi\left(v, \zeta_{i j}\right)\right] x^{\epsilon}(v) \nu_{j}^{i}(v) I_{\left\{\bar{\alpha}^{\epsilon}(v)=i\right\}} d v\right. \\
& +\sum_{i=1}^{\ell} \sum_{j=1}^{m_{i}} \int_{t}^{t+s} f_{x}^{0}\left(x^{\epsilon}(v), \zeta_{i j}\right)^{T} \\
& \left.\quad \times\left[A\left(\zeta_{i j}\right)-B\left(\zeta_{i j}\right) \Psi\left(v, \zeta_{i j}\right)\right] x^{\epsilon}(v)\left[I_{\left\{\alpha^{\epsilon}(v)=\zeta_{i j}\right\}}-\nu_{j}^{i}(v) I_{\left\{\bar{\alpha}^{\epsilon}(v)=i\right\}}\right] d v\right\} .
\end{aligned}
$$

By the weak convergence of $\left(x^{\epsilon}(\cdot), \bar{\alpha}^{\epsilon}(\cdot)\right)$ to $(x(\cdot), \bar{\alpha}(\cdot))$, the Skorohod representation, the boundedness of $f(\cdot)$, and Lemma 2.5 , for each $i=1, \ldots, 1$ and $j=1, \ldots, m_{i}$, 


$$
\begin{gathered}
E\left|\int_{t}^{t+s} f_{x}^{0}\left(x^{\epsilon}(v), \zeta_{i j}\right)^{T}\left[A\left(\zeta_{i j}\right)-B\left(\zeta_{i j}\right) \Psi\left(v, \zeta_{i j}\right)\right] x^{\epsilon}(v)\left(I_{\left\{\alpha^{\epsilon}(v)=\zeta_{i j}\right\}}-\nu_{j}^{i}(v) I_{\left\{\bar{\alpha}^{\epsilon}(v)=i\right\}}\right) d v\right|^{2} \\
\rightarrow 0 \text { as } \epsilon \rightarrow 0 .
\end{gathered}
$$

Thus the term in the second double sums on the right in (3.10) goes to 0. As for the term in the first double sums on the right in (3.10), since $\bar{\alpha}^{\epsilon}(\cdot)$ converges weakly to $\bar{\alpha}(\cdot), I_{\left\{\bar{\alpha}^{\epsilon}(v)=i\right\}}$ converges to $I_{\{\bar{\alpha}(v)=i\}}$. Then, by virtue of the Skorohod representation, as $\epsilon \rightarrow 0$,

$$
\begin{aligned}
& E \prod_{\iota=1}^{\kappa} h_{\iota}\left(x^{\epsilon}\left(t_{\iota}\right), \bar{\alpha}^{\epsilon}\left(t_{\iota}\right)\right) \\
& \times\left[\sum_{i=1}^{\ell} \sum_{j=1}^{m_{i}} \int_{t}^{t+s} f_{x}\left(x^{\epsilon}(v), i\right)^{T}\left[A\left(\zeta_{i j}\right)-B\left(\zeta_{i j}\right) \Psi\left(v, \zeta_{i j}\right)\right] x^{\epsilon}(v) \nu_{j}^{i}(v) I_{\left\{\bar{\alpha}^{\epsilon}(v)=i\right\}} d v\right] \\
& \rightarrow E \prod_{\iota=1}^{\kappa} h_{\iota}\left(x\left(t_{\iota}\right), \bar{\alpha}\left(t_{\iota}\right)\right) \\
& \times\left[\sum_{i=1}^{\ell} \sum_{j=1}^{m_{i}} \int_{t}^{t+s} f_{x}(x(v), i)^{T}\left[A\left(\zeta_{i j}\right)-B\left(\zeta_{i j}\right) \Psi\left(v, \zeta_{i j}\right)\right] x(v) \nu_{j}^{i}(v) I_{\{\bar{\alpha}(v)=i\}} d v\right] \\
& =E \prod_{i=1}^{\kappa} h_{\iota}\left(x\left(t_{\iota}\right), \bar{\alpha}\left(t_{\iota}\right)\right)\left[\int_{t}^{t+s} f_{x}(x(v), i)^{T} \overline{A(\bar{\alpha}(v))-B(\bar{\alpha}(v)) \Psi(v, \bar{\alpha}(v))} x(v) d v\right],
\end{aligned}
$$

where $\overline{A(\bar{\alpha}(v))-B(\bar{\alpha}(v)) \Psi(v, \bar{\alpha}(v))}$ is defined by (2.22).

Next, for the covariance of the diffusion (the second order term in the operator), we have

$$
\begin{array}{r}
E \prod_{\iota=1}^{\kappa} h_{\iota}\left(x^{\epsilon}\left(t_{\iota}\right), \bar{\alpha}^{\epsilon}\left(t_{\iota}\right)\right) \\
\times\left(\int_{t}^{t+s} x^{\epsilon}(v)^{T}\left[C\left(\zeta_{i j}\right)-D\left(\zeta_{i j}\right) \Psi\left(v, \zeta_{i j}\right)\right]^{T} f_{x x}^{0}\left(x^{\epsilon}(v), \zeta_{i j}\right)\right. \\
\left.\times\left[C\left(\zeta_{i j}\right)-D\left(\zeta_{i j}\right) \Psi\left(v, \zeta_{i j}\right)\right] x^{\epsilon}(v) I_{\left\{\alpha^{\epsilon}(v)=\zeta_{i j}\right\}} d v\right) \\
=E \prod_{\iota=1}^{\kappa} h_{\iota}\left(x^{\epsilon}\left(t_{\iota}\right), \bar{\alpha}^{\epsilon}\left(t_{\iota}\right)\right) \\
\times\left(\int_{t}^{t+s} x^{\epsilon}(v)^{T}\left[C\left(\zeta_{i j}\right)-D\left(\zeta_{i j}\right) \Psi\left(v, \zeta_{i j}\right)\right]^{T} f_{x x}\left(x^{\epsilon}(v), i\right)\right.
\end{array}
$$




$$
\begin{gathered}
\times\left[C\left(\zeta_{i j}-D\left(\zeta_{i j}\right) \Psi\left(v, \zeta_{i j}\right)\right] x^{\epsilon}(v) \nu_{j}^{i}(v) I_{\left\{\bar{\alpha}^{\epsilon}(v)=i\right\}} d v\right. \\
+\int_{t}^{t+s} x^{\epsilon}(v)^{T}\left[C\left(\zeta_{i j}\right)-D\left(\zeta_{i}\right) \Psi\left(v, \zeta_{i}\right)\right]^{T} f_{x x}^{0}\left(x^{\epsilon}(v), \zeta_{i j}\right)\left[C\left(\zeta_{i j}\right)-D\left(\zeta_{i j}\right) \Psi\left(v, \zeta_{i j}\right)\right] x^{\epsilon}(v) \\
\left.\left.\times\left[I_{\left\{\alpha^{\epsilon}(v)\right.}=\zeta_{i j}\right\}-\nu_{j}^{i}(v) I_{\left\{\bar{\alpha}^{\epsilon}(v)=i\right\}}\right] d v\right)
\end{gathered}
$$

and

$$
\begin{gathered}
E \prod_{\iota=1}^{\kappa} h_{\iota}\left(x^{\epsilon}\left(t_{\iota}\right), \bar{\alpha}^{\epsilon}\left(t_{\iota}\right)\right)\left(x^{\epsilon}(v)^{T}\left[C\left(\zeta_{i j}\right)-D\left(\zeta_{i j}\right) \Psi\left(v, \zeta_{i j}\right)\right]^{T} f_{x x}^{0}\left(x^{\epsilon}(v), \zeta_{i j}\right)\right. \\
\left.\left.\times\left[C\left(\zeta_{i j}\right)-D\left(\zeta_{i j}\right) \Psi\left(v, \zeta_{i j}\right)\right] x^{\epsilon}(v)\left[I_{\left\{\alpha^{\epsilon}(v)\right.}=\zeta_{i j}\right\}-\nu_{j}^{i}(v) I_{\left\{\bar{\alpha}^{\epsilon}(v)=i\right\}}\right] d v\right) \\
\rightarrow 0 \text { as } \epsilon \rightarrow 0 .
\end{gathered}
$$

Thus, we need only consider the remaining terms in (3.12). It follows that as $\epsilon \rightarrow 0$,

$$
\begin{gathered}
E \prod_{\iota=1}^{\kappa} h_{\iota}\left(x^{\epsilon}\left(t_{\iota}\right), \bar{\alpha}^{\epsilon}\left(t_{\iota}\right)\right)\left(\sum_{i=1}^{\ell} \sum_{j=1}^{m_{i}} \int_{t}^{t+s} x^{\epsilon}(v)^{T}\left[C\left(\zeta_{i j}\right)-D\left(\zeta_{i j}\right) \Psi\left(v, \zeta_{i j}\right)\right]^{T} f_{x x}\left(x^{\epsilon}(v), i\right)\right. \\
\times\left[C\left(\zeta_{i j}\right)-D\left(\zeta_{i j}\right) \Psi\left(v, \zeta_{i j}\right)\right] x^{\epsilon}(v)\left[I_{\left\{\alpha^{\epsilon}(v)=\zeta_{i j}\right\}} d v\right) \\
\rightarrow E \prod_{\iota=1}^{\kappa} h_{\iota}\left(x\left(t_{\iota}\right), \bar{\alpha}\left(t_{\iota}\right)\right)\left(\sum_{i=1}^{\ell} \sum_{j=1}^{m_{i}} \int_{t}^{t+s} x(v)^{T}\left[C\left(\zeta_{i j}\right)-D\left(\zeta_{i j}\right) \Psi\left(v, \zeta_{i j}\right)\right]^{T} f_{x x}(x(v), i)\right. \\
\times\left[C\left(\zeta_{i j}-D\left(\zeta_{i j}\right) \Psi\left(v, \zeta_{i j}\right)\right] x(v) \nu_{j}^{i}(v) I_{\{\bar{\alpha}(v)=i\}} d v\right) \\
\left.=E \prod_{\iota=1}^{\kappa} h_{\iota}\left(x\left(t_{\iota}\right), \bar{\alpha}\left(t_{\iota}\right)\right) \int_{t}^{t+s} \bar{x}(v)^{T} \Sigma(v, \bar{\alpha}(v))^{T} f_{x x}(x(v), i) \Sigma(v)\right) x(v) d v . \quad(3.13)
\end{gathered}
$$

For the jump term, the block diagonal structure of $\widetilde{Q}(t)$ and definition (3.6) imply that

$$
\widetilde{Q}(t) f^{0}(x, \cdot)(\alpha)=0 \text { for each } x \text { and each } \alpha \in \mathcal{M}
$$

so

$$
Q^{\epsilon}(t) f^{0}(x, \cdot)(\alpha)=\widehat{Q}(t) f^{0}(x, \cdot)(\alpha) .
$$

Using (2.1) and (3.14), 


$$
\begin{gathered}
E \prod_{\iota=1}^{\kappa} h_{\iota}\left(x^{\epsilon}\left(t_{\iota}\right), \bar{\alpha}^{\epsilon}\left(t_{\iota}\right)\right)\left[\int_{t}^{t+s} Q^{\epsilon}(v) f^{0}\left(x^{\epsilon}(v), \cdot\right)\left(\zeta_{i j}\right) d v\right] I_{\left\{\alpha^{\epsilon}(v)=\zeta_{i j}\right\}} \\
=E \prod_{\iota=1}^{\kappa} h_{\iota}\left(x^{\epsilon}\left(t_{\iota}\right), \bar{\alpha}^{\epsilon}\left(t_{\iota}\right)\right)\left[\int_{t}^{t+s} \widehat{Q}(v) f\left(x^{\epsilon}(v), \cdot\right)(i) \nu_{j}^{i}(v) I_{\left\{\bar{\alpha}^{\epsilon}(v)=i\right\}} d v\right] \\
+E \prod_{\iota=1}^{\kappa} h_{\iota}\left(x^{\epsilon}\left(t_{\iota}\right), \bar{\alpha}^{\epsilon}\left(t_{\iota}\right)\right)\left[\int_{t}^{t+s} \widehat{Q}(v) f^{0}\left(x^{\epsilon}(v), \cdot\right)\left(\zeta_{i j}\right)\left[I_{\left\{\alpha^{\epsilon}(v)=\zeta_{i j}\right\}}-\nu_{j}^{i}(v) I_{\left\{\bar{\alpha}^{\epsilon}(v)=i\right\}}\right] d v\right] .
\end{gathered}
$$

Again, using Lemma 2.5,

$$
\begin{gathered}
E \mid \int_{t}^{t+s} \widehat{Q}(v) f^{0}\left(x^{\epsilon}(v), \cdot\right)\left(\zeta_{i j}\right)\left[I_{\left\{\alpha^{\epsilon}(v)=\zeta_{i j}\right\}}-\left.\nu_{j}^{i}\left(v I_{\left\{\bar{\alpha}^{\epsilon}(v)=i\right\}}\right]\right|^{2}\right. \\
\rightarrow 0 \text { as } \epsilon \rightarrow 0
\end{gathered}
$$

Moreover,

$$
\begin{aligned}
& E \mid \int_{t}^{t+s}\left[\widehat{Q}(v) f\left(x^{\epsilon}(v), \cdot\right)(i) \nu_{j}^{i}(v) I_{\left\{\bar{\alpha}^{\epsilon}(v) i\right\}}\right. \\
&\left.-\bar{Q}(v) f\left(x^{\epsilon}(v), \cdot\right)(i) \nu_{j}^{i}(v) I_{\left\{\bar{\alpha}^{\epsilon}(v)=i\right\}}\right]\left.d v\right|^{2} \\
& \rightarrow 0 \text { as } \epsilon \rightarrow 0 .
\end{aligned}
$$

Combining all the estimates above, we find that $\left(x^{\epsilon}(\cdot), \bar{\alpha}^{\epsilon}(\cdot)\right)$ converges weakly to $(x(\cdot), \bar{\alpha}(\cdot))$ such that $(x(\cdot), \bar{\alpha}(\cdot))$ is the solution of the martingale problem which has a unique solution with operator (3.4). The desired result thus follows.

\subsection{Convergence of the Cost Function}

We next demonstrate the following result about the convergence of the cost function.

Theorem 3.4: Under the conditions of Theorem 3.3, as $\epsilon \rightarrow 0, J^{\epsilon}(x, \alpha)$ converges to $J(x, \bar{\alpha})$.

Proof: By virtue of weak convergence, the Skorohod representation, and the dominated convergence theorem, it is easily seen that for the terminal cost

$$
E x^{\epsilon}\left(T_{0}\right)^{T} H x^{\epsilon}\left(T_{0}\right) \rightarrow E x\left(T_{0}\right)^{T} H x\left(T_{0}\right) \text { as } \epsilon \rightarrow 0 .
$$

As for the running cost,

$$
\int_{0}^{T_{0}} x^{\epsilon}(t)^{T} M\left(\alpha^{\epsilon}(t)\right) x^{\epsilon}(t) d t=\sum_{i=1}^{\ell} \sum_{j=1}^{m_{i}} \int_{0}^{T_{0}} x^{\epsilon}(t)^{T} M\left(\zeta_{i j}\right) x^{\epsilon}(t) I_{\left\{\alpha^{\epsilon}(t)=\zeta_{i j}\right\}} d t
$$




$$
\begin{gathered}
=\sum_{i=1}^{\ell} \sum_{j=1}^{m_{i}} \int_{0}^{T_{0}} x^{\epsilon}(t)^{T} M\left(\zeta_{i j}\right) x^{\epsilon}(t) \nu_{j}^{i}(t) I_{\left\{\bar{\alpha}^{\epsilon}(t)=i\right\}} d t \\
+\sum_{i=1}^{\ell} \sum_{j=1}^{m_{i}} \int_{0}^{T_{0}} x^{\epsilon}(t)^{T} M\left(\zeta_{i j}\right) x^{\epsilon}(t)\left[I_{\left\{\alpha^{\epsilon}(t)=\zeta_{i j}\right\}}-\nu_{j}^{i}(t) I_{\left\{\bar{\alpha}^{\epsilon}(t)=i\right\}}\right] d t .
\end{gathered}
$$

In the proof of Lemma 2.5, by replacing $g(x)$ by $x$ and carrying out similar estimates, the last term in (3.17) becomes

$$
\begin{gathered}
E\left|\sum_{i=1}^{\ell} \sum_{j=1}^{m_{i}} \int_{0}^{T_{0}} x^{\epsilon}(t)^{T} M\left(\zeta_{i j}\right) x^{\epsilon}(t)\left[I_{\left\{\alpha^{\epsilon}(t)=\zeta_{i j}\right\}}-\nu_{j}^{i}(t) I_{\left\{\bar{\alpha}^{\epsilon}(t)=i\right\}}\right] d t\right|^{2} \\
\rightarrow 0 \text { as } \epsilon \rightarrow 0 .
\end{gathered}
$$

For the term in the second line of (3.17), the weak convergence of $\bar{\alpha}^{\epsilon}(\cdot)$ to $\bar{\alpha}(\cdot)$ and the Skorohod representation imply that $I_{\left\{\bar{\alpha}^{\epsilon}(t)=i\right\}} \rightarrow I_{\{\bar{\alpha}(t)=i\}}$, and that, as $\epsilon \rightarrow 0$,

$$
\begin{gathered}
E \sum_{i=1}^{\ell} \sum_{j=1}^{m_{i}} \int_{0}^{T_{0}} x^{\epsilon}(t)^{T} M\left(\zeta_{i j}\right) x^{\epsilon}(t) \nu_{j}^{i}(t) I_{\left\{\bar{\alpha}^{\epsilon}(t)=i\right\}} d t \\
\rightarrow E \sum_{i=1}^{\ell} \sum_{j=1}^{m_{i}} \int_{0}^{T_{0}} x(t)^{T} M\left(\zeta_{i j}\right) x(t) \nu_{j}^{i}(t) I_{\{\bar{\alpha}(t)=i\}} d t \\
=E \int_{0}^{T_{0}} x(t)^{T} \bar{M}(\bar{\alpha}(t)) x(t) d t .
\end{gathered}
$$

Similarly, as $\epsilon \rightarrow 0$,

$$
\begin{gathered}
E \int_{0}^{T_{0}} x^{\epsilon}(t)^{T} \Psi\left(t, \alpha^{\epsilon}(t)\right)^{T} N\left(\alpha^{\epsilon}(t)\right)^{T} N\left(\alpha^{\epsilon}(t)\right) \Psi\left(t, \alpha^{\epsilon}(t)\right) x^{\epsilon}(t) d t \\
\rightarrow E \sum_{i=1}^{\ell} \sum_{j=1}^{m_{i}} \int_{0}^{T_{0}} x(t)^{T} \Psi\left(t, \zeta_{i j}\right)^{T} N\left(\zeta_{i j}\right) \Psi\left(t, \zeta_{i j}\right) x(t) \nu_{j}^{i}(t) I_{\{\bar{\alpha}(t)=i\}} d t \\
=E \int_{0}^{T_{0}} x(t)^{T} \overline{\Psi(t, \bar{\alpha}(t))^{T} N(\bar{\alpha}(t)) \Psi(t, \bar{\alpha}(t))} x(t) d t .
\end{gathered}
$$

Finally, as in the argument given in [12, Section 9.4], for any $\alpha=s_{i j} \in \mathcal{M}$, the limit of the cost function depends only on $i \in \overline{\mathcal{M}}$. This establishes our assertion.

\section{Inclusion of Transient States}


In this section, we present the results for the generator $\widetilde{Q}(t)$ corresponding to the Markov chains that include additional transient states. Suppose that the dominating part of the generator $\widetilde{Q}(t)$ has the form

$$
\widetilde{Q}(t)=\left(\begin{array}{ccccc}
\widetilde{Q}^{1}(t) & & & & \\
& \widetilde{Q}^{2}(t) & & & \\
& & \ddots & & \\
\widetilde{Q}_{*}^{1}(t) & \widetilde{Q}_{*}^{2}(t) & & \widetilde{Q}_{*}^{l}(t) & \widetilde{Q}_{*}(t)
\end{array}\right) .
$$

The state space $\mathcal{M}$ can now be decomposed as

$$
\begin{gathered}
\mathcal{M}=\left\{\zeta_{11}, \ldots, \zeta_{1 m_{1}}\right\} \cup \ldots \cup\left\{\zeta_{l 1}, \ldots, \zeta_{l m_{l}}\right\} \cup\left\{\zeta_{* 1}, \ldots, \zeta_{* m_{*}}\right\} \\
=\mathcal{M}_{1} \cup \ldots \cup \mathcal{M}_{l} \cup \mathcal{M}_{*},
\end{gathered}
$$

where $\mathcal{M}_{i}=\left\{\zeta_{i 1}, \ldots, \zeta_{i m_{i}}\right\}$, for $i=1, \ldots, \ell$, denote the states corresponding to the states belonging to the $i$ th weakly irreducible class, and $\mathcal{M}_{i}=\left\{\zeta_{* 1}, \ldots, \zeta_{* m_{*}}\right\}$ denotes the set of transient states. In lieu of (A1), assume $\left(\mathrm{A}^{\prime}\right)$ below.

$\left(\mathbf{A 1}^{\prime}\right)$ (A1) holds. Furthermore, $\widetilde{Q}_{*}(t)$ is Hurwitz (i.e., all eigenvalues have negative real parts.) For $i=1, \ldots, \ell$, there exist constant matrices $G_{*}^{i} \in \mathbb{R}^{m_{*} \times m_{i}}$, $G_{*} \in \mathbb{R}^{m_{*} \times m_{*}}$, and a matrix-valued function $\widetilde{B}(t) \in \mathbb{R}^{m_{*} \times m_{*}}$ such that $\widetilde{B}(\cdot)$ is Lipschitz continuous and,

$$
\widetilde{Q}_{*}^{k}(t)=\widetilde{B}(t) G_{*}^{*} \text { and, } \widetilde{Q}_{*}(t)=\widetilde{B}(t) G_{*} .
$$

Motivation for using condition $\left(\mathrm{A}^{\prime}\right)$ for the transient states can be found in [14]. Define

$$
a_{m_{i}}(t)=-\widetilde{Q}_{*}^{-1}(t) \widetilde{Q}_{*}^{i}(t) \mathbb{I}_{m_{i}} \text { for } i=1, \ldots, \ell .
$$

Denote the $j$ the component of $a_{m_{i}}(t)$ by $a_{m_{i, j}}(t)$. Then it can be shown, as in [13, 14], for each $t \in\left[0, T_{0}\right]$, and each $i=1, \ldots, \ell, j=1, \ldots, m_{*}, a_{m_{i, j}}(t) \geq 0$ and $\sum_{i=1}^{l} a_{m_{i, j}}(t)=1$, i.e., the $a_{m_{i, j}}(t)$ are probabilities. In fact, $a_{m_{i, j}}(t)$ is the transition probability from the transient state $j$ to the weakly irreducible class $\mathcal{M}_{i}$. Furthermore, condition (A1') implies that $a_{m_{i}}(t)=a_{m_{i}}$ is independent of $t$. To define an aggregated process, we need to introduce $U$, a random variable uniformly distributed on $[0,1]$ that is independent of $\alpha^{\epsilon}(\cdot)$. For each $j=1, \ldots, m_{*}$, define an integer-valued random variable $U_{j}$ by

$$
\begin{aligned}
U_{j}= & I_{\left\{0 \leq U \leq a_{m_{1}, j}\right\}}+2 I_{\left\{a_{m_{1}, j}<U \leq a_{m_{1}, j}+a_{m_{2}, j}\right\}} \\
& +\ldots+l I_{\left\{a_{m_{1}, j}+\ldots+a_{m_{\ell-1}, j}<U \leq 1\right\}} .
\end{aligned}
$$

Corresponding to each $i=1, \ldots, \ell$, we combine all the $m_{i}$ states of $\mathcal{M}_{i}$ into one state and define

$$
\bar{\alpha}^{\epsilon}(t)=\left\{\begin{array}{cc}
i, & \text { if } \alpha^{\epsilon}(t) \in \mathcal{M}_{i} \\
U_{j}, & \text { if } \alpha^{\epsilon}(t)=s_{* j} .
\end{array}\right.
$$


In this case, it can be shown, as in [14], the probability that the Markov chain is in the transient class goes to 0 . Moreover, we can still obtain the weak convergence of $\bar{\alpha}^{\epsilon}(\cdot)$ to $\bar{\alpha}(\cdot)$, where $\bar{\alpha}(\cdot)$ is a continuous-time Markov chain generated by

$$
\bar{Q}(t)=\operatorname{diag}\left(\nu^{1}(t), \ldots, \nu^{l}(t)\right)\left(\widehat{Q}^{11}(t) \operatorname{diag}\left(\mathbb{I}_{m_{1}}, \ldots, \mathbb{I}_{m_{\ell}}\right)+\widehat{Q}^{12}(t)\left(a_{m_{1}}, \ldots, a_{m_{\ell}}\right)\right)
$$

using the partition

$$
\widehat{Q}(t)=\left(\begin{array}{ll}
\widehat{Q}^{11}(t) & \widehat{Q}^{12}(t) \\
\widehat{Q}^{21}(t) & \widehat{Q}^{22}(t)
\end{array}\right)
$$

with $\quad \widehat{Q}^{11}(t) \in \mathbb{R}^{\left(m-m_{*}\right) \times\left(m-m_{*}\right)}, \quad \widehat{Q}^{12}(t) \in \mathbb{R}^{\left(m-m_{*}\right) \times m_{*}}, \quad \widehat{Q}^{21}(t) \in \mathbb{R}^{m_{*} \times\left(m-m_{*}\right)}, \quad$ and $\widehat{Q}^{22}(t) \in \mathbb{R}^{m_{*} \times m_{*}}$. In addition, similar to [16], to obtain asymptotic optimal controls of the underlying systems, one can ignore the transient states. We obtain the following results.

Theorem 4.1: Assume $\left(A 1^{\prime}\right)$ and $(A 2)$. Then the conclusions of Theorem 3.1, Theorem 3.3 and Theorem 3.4 continue to hold.

\section{Further Remarks}

In the previous sections, we demonstrated convergence of the original problem $\mathcal{P}^{\epsilon}$. Compared with the results obtained in [16], we treated nonstationary Markov chains and indefinite control weights. As far as the techniques are concerned, we have used a purely probabilistic approach via the weak convergence method and martingale averaging formulation in lieu of solving HJB equations.

The significance of this study is that the Markov chain involved in $\mathcal{P}^{\epsilon}$ usually has a large state space, whereas the number of states of the Markov chain in the limit problem $\mathcal{P}^{0}$ is substantially reduced. This is achieved by aggregating the states in each weakly irreducible class $\mathcal{M}_{i}$ into one state so the total number of states in the limit process $\bar{\alpha}(\cdot)$ becomes $\ell$. To solve the original problem via a dynamic programming approach, one needs to solve a system of Riccati equations with the total number of equations being $m$; whereas in the limit problem, the total number of equations to be solved is reduced to $\ell$. If $\ell \ll m$, the amount of effort is substantially reduced.

\section{References}

[1] Ackerman, J., Parameter space design of robust control systems, IEEE Trans. Automat. Control AC-25 (1980), 1058-1072.

[2] Chen, S., Li, X., and Zhou, X.Y., Stochastic linear quadratic regulators with indefinite control weight costs, SIAM J. Control Optim. 36 (1998), 1685-1702.

[3] Chen, S. and Yong, J., Stochastic linear quadratic optimal control problems, Appl. Math. Optim. 43 (2001), 21-45.

[4] Chen, S. and Yong, J., Stochastic linear quadratic optimal control problems with random coefficients, Chinese Ann. Math. 21B (2000), 323-338.

[5] Etheir, S.N. and Kurtz, T.G., Markov Processes: Characterization and Convergence, J. Wiley, New York 1986.

[6] Il'in, A., Khasminskii, R., and Yin, G., Asymptotic expansions of solutions of integrodifferential equations for transition densities of singularly perturbed switching diffusions: Rapid switchings, J. Math. Anal. Appl. 238 (1999), 516-539. 
[7] Ji, Y. and Chizeck, H.J., Jump linear quadratic Gaussian control in continuous-time, IEEE Trans. Automat. Control AC-37 (1992), 1884-1892.

[8] Mariton, M., Robust jump linear quadratic control: A mode stabilizing solution, IEEE Trans. Automat. Control. AC-30 (1985), 1145-1147.

[9] Kushner, H.J., Weak Convergence Methods and Singularly Perturbed Stochastic Control and Filtering Problems, Birkhäuser, Boston 1990.

[10] Simon, H.A., Models of Discovery and Other Topics in the Methods of Science, D. Reidel Publ. Co., Boston 1977.

[11] Sethi, S.P. and Zhang, Q., Hierarchical Decision Making in Stochastic Manufacturing Systems, Birkhäuser, Boston 1994.

[12] Yin, G. and Zhang, Q., Continuous-time Markov Chains and Applications: A Singular Perturbation Approach, Springer-Verlag, New York 1998.

[13] Yin, G., Zhang, Q., and Badowski, G., Asymptotic properties of a singularly perturbed Markov chain with inclusion of transient states, Ann. Appl. Probab. 10 (2000), 549-572.

[14] Yin, G., Zhang, Q., and Badowski, G., Singularly perturbed Markov chains: Convergence and aggregation, J. Multivariate Anal. 72 (2000), 208-229.

[15] Yong, J. and Zhou, X.Y., Stochastic Controls: Hamiltonian Systems and HJB Equations, Springer-Verlag, New York 1999.

[16] Zhang, Q. and Yin, G., On nearly optimal controls of hybrid LQG problems, IEEE Trans. Automat. Control 44 (1999), 2271-2282. 


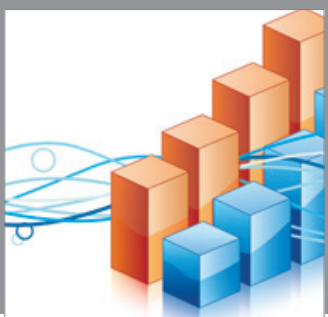

Advances in

Operations Research

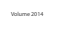

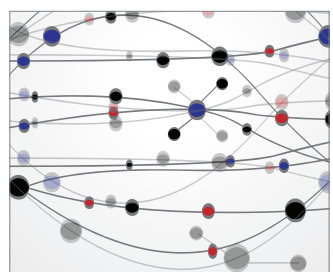

\section{The Scientific} World Journal
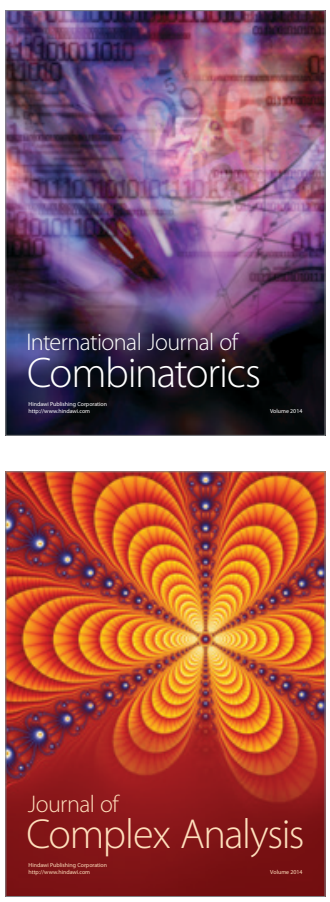

International Journal of

Mathematics and

Mathematical

Sciences
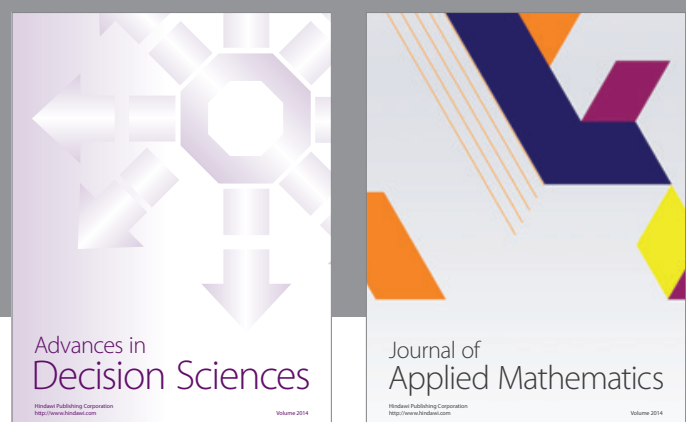

Journal of

Applied Mathematics
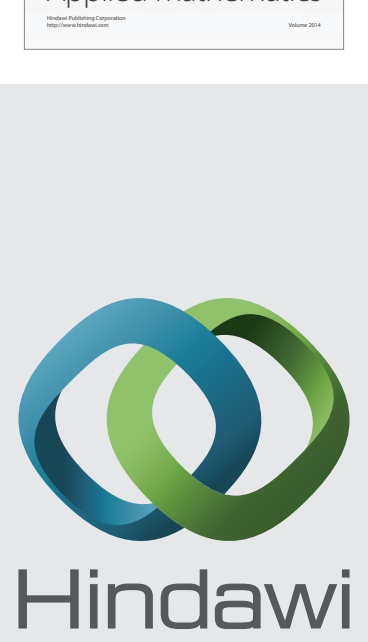

Submit your manuscripts at http://www.hindawi.com
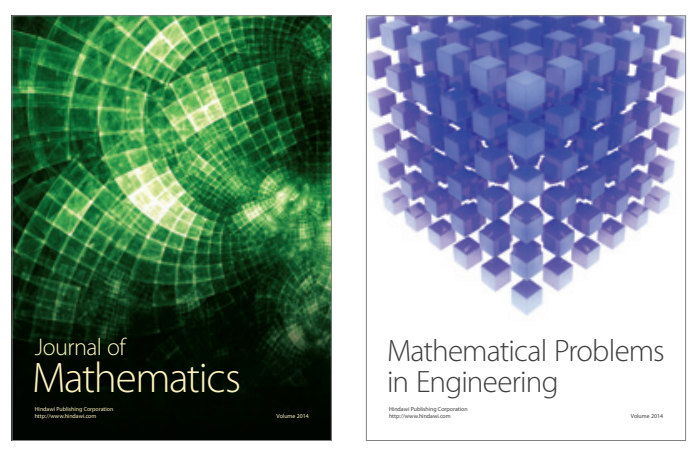

Mathematical Problems in Engineering
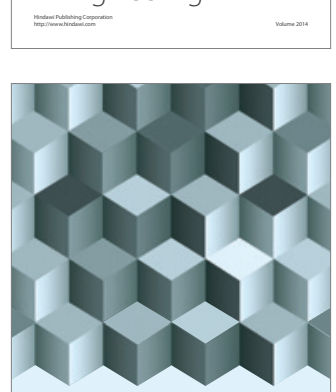

Journal of

Function Spaces
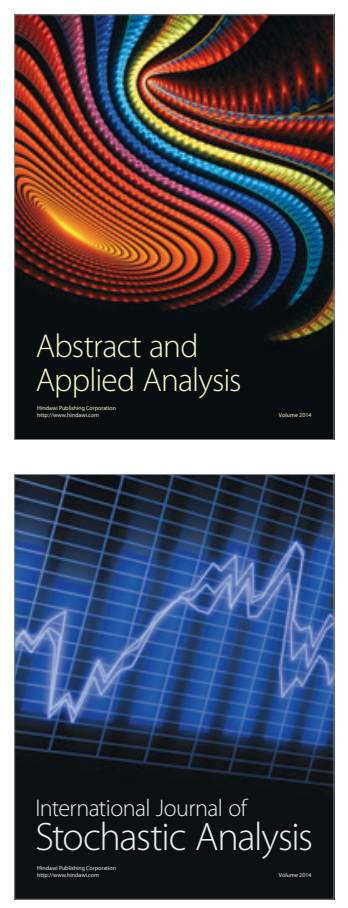

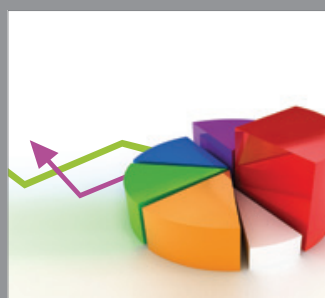

ournal of

Probability and Statistics

Promensencen
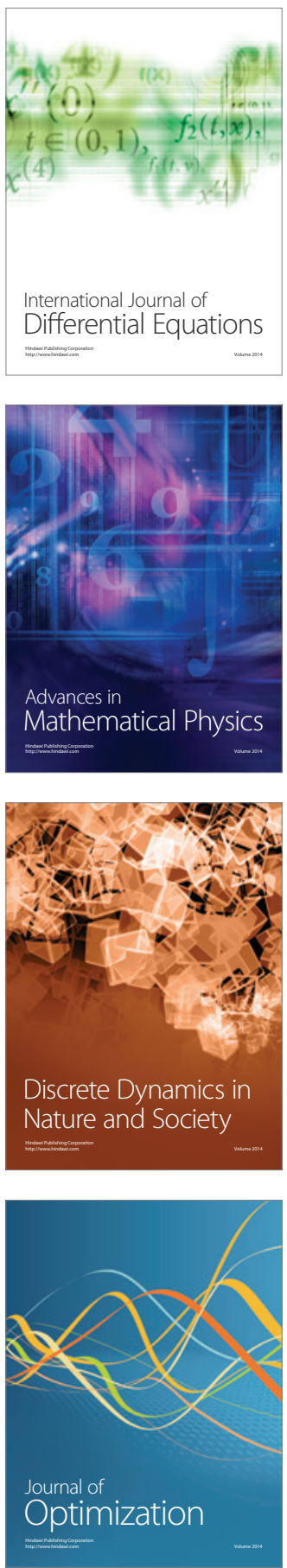